\title{
Management of Acute Puerperal Inversion of the Uterus
}

\section{INTRODUCTION}

The aim of this guideline is to provide recommendations for the management of acute puerperal inversion of the uterus, which is a rare and life threatening condition. The main reason for its high mortality rate is delay in instituting appropriate treatment, which leads to postpartum hemorrhage and rapid development of shock out of proportion to haemorrhage.

\section{DEFINITION}

It is defined as 'the turning inside out of the fundus into the uterine cavity'.

\section{PREVENTION}

Mismanagement of the third stage of labor is recognized as the main cause, although $50 \%$ have no identifiable cause. The common initiating factor seems to be a traction force on the fundus of a relaxed uterus. Proper retraction of the uterus in the third stage is the primary factor in preventing an inversion. There is no reliable data to suggest that it recurs in a future pregnancy.

The importance of the active management of the third stage could not be over-emphasized. (Please refer Section 3 of the PPH Guideline and the section on management of delayed third stage (section 4.5 in the Normal Labor Guideline for details)

\section{PATHOPHYSIOLOGY (AND CLINICAL CORRELATION)}

As the inversion progresses, the adnexae with their ligaments get drawn into the inverting uterine fundus and become increasingly stretched. This produces significant pain and vagal stimulation, leading to neurogenic shock. An inverted uterus becomes trapped within the cervix creating progressive oedema and congestion due to interruption of venous and lymphatic drainage. Oedema and congestion will increase the firmness of the inverted segment, making reduction more difficult. Interruption of the venous drainage will lead to significant haemorrhage. A partially separated placenta would add to this.

\section{CLASSIFICATION}

Although acute, subacute and chronic varieties have been described, this guideline would address only the acute variety as it is life threatening.

This occurs soon after birth, just before or after the delivery of the placenta. Three degrees of inversion have been described, depending on the level of the inverted fundus. In practice, second-degree inversion is the commonest.
The fundus has come past the cervical as, but is still within the vagina.

\section{CLINICAL PRESENTATION AND DIAGNOSIS Prompt diagnosis is vital}

The key to diagnosis is awareness and a high degree of suspicion.

The following are early warnings:

- A degree of shock that is out of proportion to overt blood loss

- A retained placenta

- Placenta delivered but 'with some difficulty'

- Severe, sustained unexplained pain in the third stage.

In this situation:

- Feel for the fundus. If absent or 'cupped', acute inversion is probable diagnosis;

- Confirm by a vaginal examination:

- Look for a hard mass which looks and feels like a huge ulcerated fibroid polyp (sometimes described as a foetal head);

- The cervix is not to be seen or felt in the normal position, instead it could be felt as a ring around the base of the 'mass';

- In incomplete cases, the inverted fundus may be felt through the cervical canal in the lower uterine cavity.

\section{MANAGEMENT}

\subsection{General measures}

Early diagnosis is vital. Treat it as a life-threatening emergency.

First attempts at reduction should be made at the place where it is diagnosed, without moving to theatre.

If these attempts fail, move to theatre and give a general anesthetic without delay (see section 7.2.4).

Early involvement of experienced personnel and teamwork are absolutely essential.

Treat shock aggressively, not forgetting the neurogenic element.

Provide adequate pain relief.

Replace the blood loss, which could be considerable, especially if the placenta has partially or completely separated.

Do not attempt to remove the placenta, if still attached. 


\subsection{Repositioning the uterus}

Reposition the uterus as soon as possible; the sooner it is done the easier and better. It reverses the shock and reduces $\mathrm{PPH}$

\section{Non-surgical methods}

\subsubsection{Manual replacement of uterus}

(Johnson's maneuver)

The operator introduces two thirds of his forearm in to the vagina and extends the hand at the wrist to place the palm on the inverted fundus and fingertips at the uterocervical junction. Lifting the uterus above the level of the umbilicus creates adequate tension for the cervical ring to dilate and for the fundus to revert to its normal position.

This could be helped by 'working the fingers up' gradually from the cervical ring towards the fundus, with gentle but persistent pressure applied.

Where the uterus is too hard to respond, consider tocolytics (see below).

Once reduced, hold the fundus in place for a few minutes (making a fist inside the uterus with upward pressure on the fundus helps).

Administer uterotonics (Ergometrine $0.25 \mathrm{mg}$ i.v. or oxytocin 5-10 IU i.v followed by oxytocin infusion at the rate of $10 \mathrm{IU}$ per hour), whilst the hand is still inside. When the uterus begins to contract, slowly remove the hand.

This manouvre is possible only soon after the event, and would need adequate analgesia. Unless it is possible to administer either a general anesthetic immediately, administer pethidine $50 \mathrm{mg}$ iv slow and proceed with the maneuvres.

Give antibiotics (e.g. cephradine 19 and metronidazole 500 mg IV).

\subsubsection{Hydrostatc reduction (O'Sullivan 1945)}

Several novel and useful modifications have been made to this procedure lately, principally to circumvent the problem of inadequate water seal, which has been the major cause of failure in the past.

Insert $6 \mathrm{~cm}$ silastic ventouse cup into vagina, making sure that it is directed at the posterior vaginal fornix and not at, or cupping the fundus. Place hand at introitus to maintain seal between cup and vagina.

(Alternatively $500 \mathrm{ml}$ balloon catheter can be placed in vagina. If neither is available, use a wide tube; a standard giving set will not do)

Connect via IV giving set to a bag of warmed normal saline placed 1- 1.5 metres above the patient.

Infuse normal saline (typically 2 litres) into vagina to reduce the uterus by hydrostatic pressure. Once reduced, remove the placenta if still attached and proceed as in the previous section.
Where a balloon is used, it would be advisable to leave it for 12-24 hours to prevent re-inversion and reduce haemorrhage.

Saline embolisation and fluid overload leading to pulmonary oedema are only theoretical risks as long as one sticks to hydrostatic pressure only.

\subsubsection{Tocolytics}

Where repositioning is difficult due to retraction of the uterine muscle and the constriction of the cervical ring, tocolytics could be helpful. But given this could cause PPH, it would have to be a considered and a senior decision. They are safest given in the theatre setting.

Various preparations have been used; ideally it should be readily available, with quick onset and short duration of action.

E.g. Turbutaline $0.25 \mathrm{mg}$ i.v. slowly (not available in Sri Lanka at present);

Salbutamol $0.25 \mathrm{mg}$ in $10 \mathrm{ml}$ saline i.v. slowly;

Nitroglycerine $0.1 \mathrm{mg}$ i.v. slowly or sublingually (acts within 90 seconds)

\subsubsection{General Anaesthesia}

If the initial attempt at manual replacement fails, it is safest to move the patient to the theatre and to administer general anaesthesia. This allows for muscle relaxation, pain relief and elimination of the neurogenic contribution to the shock.

\subsubsection{Surgical methods}

If managed properly in the early stages, resort to surgery should be a rare occurrence.

\section{Huntingdon's operation}

After a laparotomy, the indrawn uterine cup is identified near the region of the cervix with the tubes and round ligaments pulled into the cup. By the use of two Allis forceps the uterus is pulled out of the constriction ring in a progressive fashion and restored to its normal position. The serosa of the uterus will invariably sustain lacerations and these are repaired with absorbable sutures.

Use of a silastic vacuum cup from above instead of Allis forceps has been shown to circumvent this problem.

\section{Haultain's operation}

In this procedure the constriction in the region of cervix is incised posteriorly using a longitudinal incision. As in the Huntingdon's method two Allis forceps are used to pull the uterus to its normal position. The incision is repaired with interrupted sutures. Uterotonics are given to maintain contraction of the uterus. 


\section{Hysterectomy}

When all the above methods fail, a hysterectomy will become the only viable option. However, it must be remembered that given the distorted anatomy, this must be undertaken by a surgeon of considerable experience.

\section{DEBRIEFING}

Although there is no evidence of a recurrence risk, it is sensible to advise the woman to deliver in a specialized Unit next time, and the third stage to be managed actively by experienced personnel. 